ARTICLE

\title{
Gain-switched semiconductor laser driven soliton microcombs
}

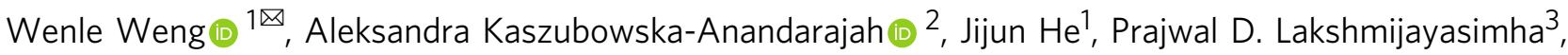 \\ Erwan Lucas ${ }^{1,4,5}$, Junqiu Liu (iD ${ }^{1}$, Prince M. Anandarajah (iD ${ }^{3 凶} \&$ Tobias J. Kippenberg (iD) ${ }^{1 凶}$
}

Dissipative Kerr soliton generation using self-injection-locked III-V lasers has enabled fully integrated hybrid microcombs that operate in turnkey mode and can access microwave repetition rates. Yet, continuous-wave-driven soliton microcombs exhibit low energy conversion efficiency and high optical power threshold, especially when the repetition frequencies are within the microwave range that is convenient for direct detection with off-the-shelf electronics. Here, by actively switching the bias current of injection-locked III-V semiconductor lasers with switching frequencies in the X-band and $\mathrm{K}$-band microwave ranges, we pulsepump both crystalline and integrated microresonators with picosecond laser pulses, generating soliton microcombs with stable repetition rates and lowering the required average pumping power by one order of magnitude to a record-setting level of a few milliwatts. In addition, we unveil the critical role of the phase profile of the pumping pulses, and implement phase engineering on the pulsed pumping scheme, which allows for the robust generation and the stable trapping of solitons on intracavity pulse pedestals. Our work leverages the advantages of the gain switching and the pulse pumping techniques, and establishes the merits of combining distinct compact comb platforms that enhance the potential of energyefficient chipscale microcombs.

\footnotetext{
${ }^{1}$ Institute of Physics, Swiss Federal Institute of Technology Lausanne (EPFL), Lausanne, Switzerland. ${ }^{2}$ CONNECT Research Centre, Dunlop Oriel House, Trinity College Dublin, Dublin, Ireland. ${ }^{3}$ Photonics Systems and Sensing Laboratory, School of Electronic Engineering, Dublin City University,

Glasnevin, Ireland. ${ }^{4}$ Present address: Time and Frequency Division, NIST, Boulder, CO, USA. ${ }^{5}$ Present address: Department of Physics, University of Colorado, Boulder, CO, USA.『email: wenle.weng@epfl.ch; prince.anandarajah@dcu.ie; tobias.kippenberg@epfl.ch
} 
D eveloped nearly four decades ago, the gain-switching technique is an elegant approach to generate optical pulse trains with semiconductor lasers ${ }^{1-3}$. By applying a microwave signal to the bias current of a semiconductor laser, coherent pulses with a repetition rate up to a few tens of gigahertz can be emitted from the gain medium, constituting optical frequency combs ${ }^{4,5}$. The simplicity, flexibility and low cost associated with this technique not only facilitate its use in classical optical telecommunications $s^{6,7}$ and secure quantum communication networks ${ }^{8-10}$ but also may lead to valuable applications in microwave photonics ${ }^{11-13}$. However, because of the intrinsic laser dynamics, the temporal durations of gain-switched laser (GSL) pulses are typically limited to a few tens of picoseconds, corresponding to narrow spectral spans of a few nanometres. Although spectral broadening with nonlinear fibre can be adopted to increase the GSL comb bandwidth ${ }^{5}$, the need for multistage optical amplification and lengthy fibres precludes the use of GSLs in integrated broadband frequency comb generation.

On a different path towards the development of fully integrated frequency combs, soliton microcombs ${ }^{14,15}$ have been revolutionising the frequency comb technology by producing femtosecond laser pulses with Kerr microresonators. Owing to the broad spectral width and the inherent low timing jitter of microresonator-dissipative Kerr solitons (DKSs), besides the demonstration of coherent communications ${ }^{16}$, soliton microcombs have also been used in system-level applications in ultrafast ranging ${ }^{17,18}$ and parallel Lidar ${ }^{19}$. In the meantime, rapid progress in the development of soliton microcombs with ultralow power threshold or with powerful pump laser diode has been achieved ${ }^{20-23}$. In particular, advanced fabrication processes have enabled low-repetition-rate $(<20 \mathrm{GHz})$ soliton excitation that is of crucial importance to integrated microwave photonics ${ }^{24-26}$. Yet, the generation of low-repetition-rate soliton microcombs with the traditional continuous-wave (CW) pumping scheme typically needs a pump power of at least a few tens of milliwatts ${ }^{25,26}$, which puts a stringent requirement on the tolerable loss of the system. To break away from the paradigm of CW pumping, the pulse-pumping technique has been demonstrated $^{27}$ and applied in the calibration of astronomical spectrographs ${ }^{28}$. Instead of filling the entire resonator with intensive laser field, the pulse-pumping technique feeds in an intracavity field of soliton pedestal that occupies only a small portion of the cavity round trip, thus substantially lowering the power threshold and increasing the conversion efficiency. To date, however, this unconventional scheme is only realised by utilising cascaded high-speed modulators ${ }^{29}$, which requires bulky and expensive microwave components. As a result, the benefit of the reduced power threshold is to a large extent cancelled out by the high optical power loss induced by the modulators and the high radiofrequency ( $\mathrm{rf}$ ) driving power.

In this work, we show that the GSL and the DKS can join together to complement each other and to bring about complex soliton dynamics. We apply the gain-switching technique on different semiconductor lasers, including a distributed feedback (DFB) laser, a Fabry-Perot (FP) laser and a discrete mode (DM) laser, to demonstrate pulse-driven soliton microcombs in two microresonator platforms, namely a crystalline magnesium fluoride $\left(\mathrm{MgF}_{2}\right)$ whispering gallery mode resonator (WGMR) with a K-band microwave $(\sim 14 \mathrm{GHz})$ repetition frequency $\left(f_{\text {rep }}\right)^{30}$ and a silicon nitride $\left(\mathrm{Si}_{3} \mathrm{~N}_{4}\right)$-integrated microresonator with an Xband microwave $f_{\text {rep }}(\sim 10 \mathrm{GHz})^{26}$. We show that the GSL pumping method allows for deterministic single-soliton excitation and substantially lowers the optical power threshold. We also investigate the critical role of the phase of the GSL pulse in supporting soliton existence. We present two effective approaches to tailor the pulse profile in order to optimise robust microcomb generation, which not only paves the way to inexpensive integrated frequency combs but also reveals the fundamental soliton dynamics in a pulse-driven scenario.

\section{Results}

Semiconductor lasers for microcomb generation. Figure 1a illustrates the principle of the GSL pumping technique. To pulsepump, a soliton microcomb, the bias current of a semiconductor laser is modulated at a frequency close to the microresonator free spectral range (FSR). Because the intrinsic linewidths of the semiconductor laser modules (without isolators) used in this study are of a few megahertz, we use a 1550-nm external cavity diode laser (ECDL) to injection-lock the semiconductor lasers, thus narrowing the linewidths of the slave lasers to $\sim 20 \mathrm{kHz}$ and ensuring the pulse-to-pulse coherence during gain switching. In the future, this external injection locking setup may be replaced with self-injection locking using backscattering from the microresonator ${ }^{31,32}$. Figure $1 \mathrm{~b}$ shows the photographs of a GSL chip and a photonic microresonator, and Fig. 1c presents the natural CW lasing spectra of the DFB laser, the FP laser and the DM laser, respectively. While the DFB laser shows single-mode lasing behaviour, two first-order side modes approximately $250 \mathrm{GHz}$ apart from the central lasing mode are visible in the spectrum. In contrast, the FP laser exhibits multimode lasing with a mode separation of $175 \mathrm{GHz}$. Here we particularly introduce the DM laser, which is essentially an FP laser with additional etched features to engineering the losses of different cavity modes to obtain single-mode lasing. With a longitudinal mode spacing of $165 \mathrm{GHz}$, the DM laser spectrum displays a high side-modesuppression ratio (SMSR) of $\sim 62 \mathrm{~dB}$. More details of the GSLs can be found in Supplementary Note 1.

Pulse driving with DFB GSL. We activate the gain switching of the DFB laser with a gain-switching frequency $\left(f_{\mathrm{gs}}\right)$ provided by a microwave synthesiser. The upper panel in Fig. 2a shows a typical GSL spectrum (average power of $1 \mathrm{~mW}$ ) with $f_{\mathrm{gs}}$ of $10 \mathrm{GHz}$ when the ECDL is injected into the central mode of the DFB laser. We perform autocorrelation measurement of the amplified GSL pulses. The autocorrelation intensity displayed in the upper panel

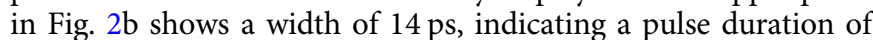
$\sim 10$ ps (assuming a Gaussian profile). The master laser is swept with decreasing frequency over a resonance of the microresonator, while $f_{\mathrm{gs}}$ is finely adjusted around the expected $f_{\text {rep }}$, in order to observe the step-like soliton signature in the transmission spectra. However, with an average GSL power of $120 \mathrm{~mW}$, which is even higher than the power level needed for CW-driven soliton generation, we do not observe steps that are wide enough for stable soliton existence. The upper panel in Fig. $2 c$ presents an example of the transmission spectra. With the optimised step width of only $\sim 2 \mathrm{MHz}$ during the laser sweep, we are not able to accurately stop the laser scan within the step range to enable soliton microcomb generation.

Surprisingly, when the ECDL is detuned from the DFB laser's central mode by more than $1 \mathrm{~nm}$, the GSL spectrum and the pulse profile change qualitatively. As can be seen from the lower panels in Fig. 2a, b, the GSL comb bandwidth increases as the spectrum show several lobes, and the autocorrelation intensity shows multiple peaks with a temporal separation of $\sim 4.7 \mathrm{ps}$, suggesting the splitting of GSL pulses. With such an offset-injection-locked state, the GSL is able to generate soliton microcombs in both the $\mathrm{Si}_{3} \mathrm{~N}_{4}$ and the $\mathrm{MgF}_{2}$ microresonators with an average pump power below $10 \mathrm{~mW}$. Figure 2d, e shows the generated $\mathrm{Si}_{3} \mathrm{~N}_{4}$ soliton microcomb with $f_{\mathrm{gs}}=9.716 \mathrm{GHz}$ and the $\mathrm{MgF}_{2}$ soliton microcomb with $f_{\mathrm{gs}}=14.093 \mathrm{GHz}$. The pump powers at a-fewmilliwatt level, to the best of our knowledge, are the lowest used 

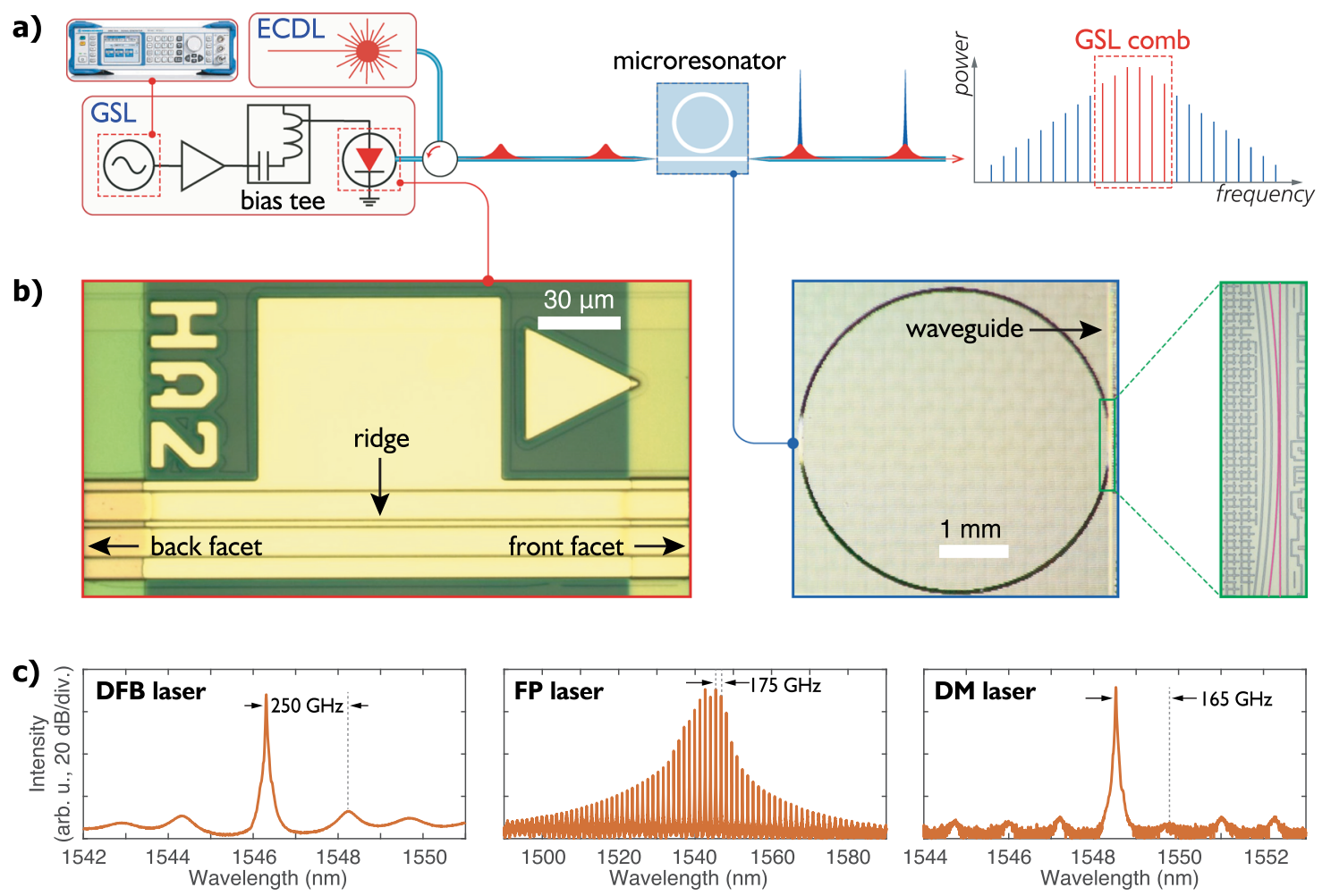

Fig. 1 Gain-switched semiconductor lasers for pulsed pumping of microcombs. a Pumping scheme for soliton generation. The bias current of an injectionlocked semiconductor laser is modulated to produce a pulse train to generate DKSs in a microresonator. A microwave synthesiser (Rohde and Schwarz SMB100A) is providing the gain-switching frequency. $\mathbf{b}$ The photographs of (left) a Fabry-Perot laser chip (middle), a $\mathrm{Si}_{3} \mathrm{~N}_{4}$ microresonator and (right) the enlarged coupling area (the microring and the waveguide are in pink for visibility). c The CW lasing spectra (i.e., without optical injection, gain switching deactivated) of the three semiconductor lasers used in this study, including (left) a distributed feedback laser, (middle) a Fabry-Perot laser and (right) a discrete mode laser.

in low-repetition-rate soliton microcombs. One should note that, due to the 3-dB lensed-fibre-to-waveguide loss, for the $\mathrm{Si}_{3} \mathrm{~N}_{4}$ microcomb, the on-chip optical power is below $5 \mathrm{~mW}$. Compared with prior works using CW pumping ${ }^{26,30}$, the GSL pumping technique lowers the required pump power by an order of magnitude without compromising the spectral width of the soliton microcomb.

In the following, we focus our effort on the $\mathrm{Si}_{3} \mathrm{~N}_{4}$ microcomb. With a GSL power of $15 \mathrm{~mW}$, we vary $f_{\mathrm{gs}}$ as we repeat the laser sweep to produce the spectrogram in Fig. $2 \mathrm{f}$. In the area outlined with dashed lines, single-soliton steps wider than $20 \mathrm{MHz}$ are observed. We set $f_{\mathrm{gs}}$ to be a constant value within this area, and perform the laser sweep for 100 consecutive times. The transmission traces are presented in the lower panel of Fig. $2 c$, showing $100 \%$ successful deterministic single-soliton excitation rate. To further confirm the pulse-pumping nature, we use an optical band-pass filter (bandwidth $\sim 0.5 \mathrm{~nm}$ ) to select the soliton microcomb at $1545.5 \mathrm{~nm}$ for $f_{\text {rep }}$ measurement. The filtered comb power is sent to a fast photodetector, whose output voltage is analysed by an electrical spectrum analyser. With the ECDL frequency scanned for $20 \mathrm{MHz}$ within the soliton step range, only a single-repetition frequency is observed, showing that $f_{\text {rep }}$ is disciplined by $f_{\mathrm{gs}}$. In contrast, with CW pumping, $f_{\text {rep }}$ is changed by $\sim 3.5 \mathrm{kHz}$ due to Raman and higher-order dispersion effects that couple the soliton repetition rate to the pump-resonance detuning ${ }^{33-35}$.

We deduce that the master laser injected at a position between the central mode and a side mode can induce the simultaneous lasing of both modes, and the interference between the two fields causes the multiple peaks in the GSL pulses. To test our hypothesis with numerical simulations, we develop a dual-lasing- mode model based on laser rate equations ${ }^{1,36}$, which are expressed as

$$
\begin{aligned}
& \frac{d N}{d t}=\frac{I_{\text {bias }}+I_{\mathrm{gs}} \sin \left(2 \pi f_{\mathrm{gs}} t\right)}{e V}-\left(\gamma_{1} N+\gamma_{2} N^{2}+\gamma_{3} N^{3}\right) \\
& -a V\left(N-N_{0}\right)\left|A_{\mathrm{GSL}}\right|^{2}+F_{\mathrm{N}}(t) \\
& \frac{d A_{1}}{d t}=\frac{1}{2}\left(1-i \alpha_{\mathrm{H}}\right)\left(a V\left(N-N_{0}\right)-\frac{1}{\tau_{\mathrm{p} 1}}\right) A_{1} \\
& +\eta \sqrt{\kappa_{\mathrm{c}}} s_{\mathrm{inj}}+F_{\mathrm{A}}(t) \\
& \frac{d A_{2}}{d t}=\frac{1}{2}\left(1-i \alpha_{\mathrm{H}}\right)\left(a V\left(N-N_{0}\right)-\frac{1}{\tau_{\mathrm{p} 2}}\right) A_{2} \\
& +i 2 \pi f_{\mathrm{sm}} A_{2}+(1-\eta) \sqrt{\kappa_{\mathrm{c}}} s_{\mathrm{inj}}+F^{\prime}(t)
\end{aligned}
$$

where $N$ is the carrier density, $A_{1}$ and $A_{2}$ are the slowly varying envelopes of the fields of the central mode and the side mode respectively, $f_{\mathrm{sm}}$ is the frequency of the side mode relative to the central mode, $\alpha_{\mathrm{H}}$ is the linewidth enhancement factor, $a$ is the differential gain, $V$ is the active volume, $N_{0}$ is the carrier density at transparency, $\kappa_{\mathrm{c}}$ is the injection coupling rate and $\left|s_{\mathrm{inj}}\right|^{2}$ is related to the injected photon flux. The carrier recombination rate is represented by $\gamma_{1} N+\gamma_{2} N^{2}+\gamma_{3} N^{3}$, showing the combined effects of the non-radiative recombination, the radiative recombination and the Auger recombination. The elementary charge is denoted by $e$. The photon density $\left|A_{\mathrm{GSL}}\right|^{2}$ in the laser-active volume is related to $A_{1}$ and $A_{2}$ with $A_{\mathrm{GSL}}=A_{1}+A_{2}$. For gainswitching operation, the gain-switching current $I_{\mathrm{gs}}$ is added to the bias current $I_{\text {bias. }}$. The spontaneous emission into the lasing field 

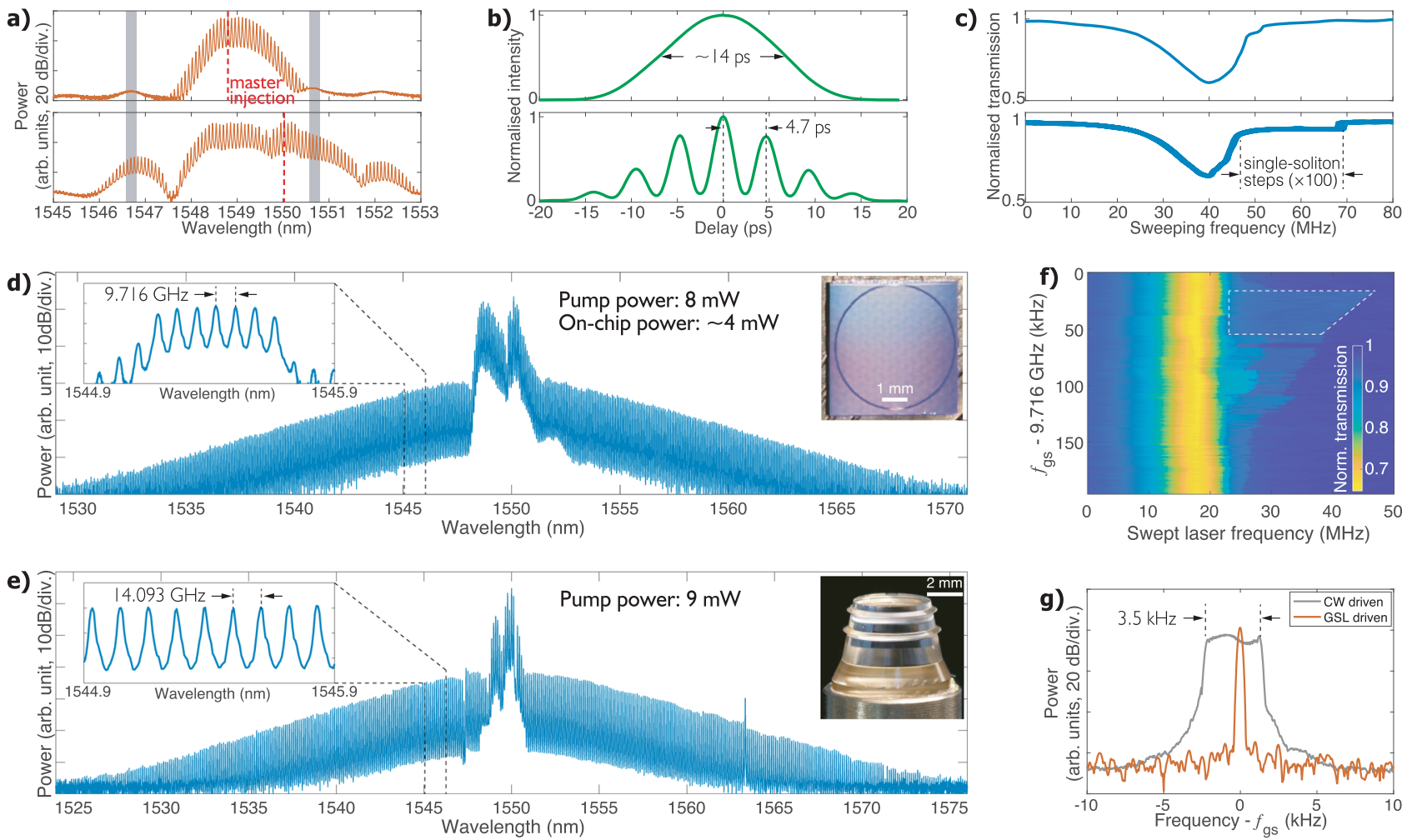

Fig. 2 Soliton microcomb generation with a gain-switched distributed feedback laser. a The GSL spectra when the master laser is injected at the central lasing position (upper panel) and an off-central-mode frequency (lower panel). The grey stripes indicate the positions of the two adjacent longitudinal modes. The red dashed lines show the wavelengths of the master laser. $\mathbf{b}$ The intensity autocorrelation of the GSL pulses when the DFB laser is centralmode-injected (upper panel) and off-centre-injected (lower panel), respectively. c Laser downsweeping transmission traces corresponding to the centralmode-injected operation (upper panel) and off-centre-injected operation (lower panel), respectively. In the lower panel, 100 consecutive sweeping traces are overlaid. The offset-centre injection locking leads to significantly longer soliton steps. $\mathbf{d}$, e The microcomb spectra of a $\mathrm{Si}_{3} \mathrm{~N}_{4} \mathrm{microring}$ resonator and an $\mathrm{MgF}_{2}$ crystalline resonator. The left-hand insets show the enlarged portions of the spectra (band-pass-filtered for the $\mathrm{Si}_{3} \mathrm{~N}_{4}$ microcomb). The right-hand insets are the photographs of the microresonators. $\mathbf{f}$ Contour plot of the laser-frequency-swept transmission as the gain-switching frequency $f_{g s}$ is varied. The trapezium outlined by dashed lines indicates the area for a robust single-soliton generation. $\mathbf{g}$ Spectra of $f_{\text {rep }}$ when the laser-resonance detuning is swept, showing that the microcomb repetition frequency is changing in CW pumping but governed by $f_{\mathrm{gs}}$ in the GSL-driven situation. The measurement resolution bandwidth (RBW) is $100 \mathrm{~Hz}$.

and the stochastic carrier recombination are denoted by the Langevin noise terms $F_{\mathrm{A}}$ and $F_{\mathrm{N}}$, respectively ${ }^{36,37}$. For simplicity, the ratio of the master laser field injected into the central mode $(\eta)$ is set to be 0.5 , and the two modes are assumed to have the same gain and linewidth enhancement factor, but different photon lifetimes. Moreover, this model neglects nonlinear interactions such as four-wave mixing. One should note that the DFB laser dynamics cannot be accurately described by this model as the modes in a DFB structure cannot be characterised with a photon lifetime like an FP mode. Nevertheless, we will show that this simplified model captures the key features of the dual-mode lasing phenomenon.

Figure $3 \mathrm{a}, \mathrm{b}$ presents the GSL spectrum and the intensity and phase (i.e., $\arg \left(A_{\mathrm{GSL}}\right)$ ) of the pulse, respectively, when the master laser is injected at the centre of the natural lasing mode (see "Methods" for the details of the simulation), showing excellent agreement with the experimental results in terms of comb bandwidth and pulse duration. Crucially, the simulated pulse exhibits a phase minimum at the intensity maximum. Since DKSs are attracted to where the intracavity pumping field has the highest phase $\mathrm{e}^{38-40}$, the GSL pulse profile is unfavourable to soliton generation because any soliton initiated by modulation instability $(\mathrm{MI})^{41}$ is drawn to the leading or the trailing edges of the pulse where the field amplitude is not adequate to support soliton existence. To confirm our analysis, we use the simulated
GSL pulse as the pumping field profile to simulate microcomb generation using the Lugiato-Lefever equation (LLE) ${ }^{42,43}$. Neglecting higher-order dispersion but including Raman effect, the LLE is written as ${ }^{35}$

$$
\begin{array}{r}
\frac{\partial A}{\partial t}+i \frac{D_{2}}{2} \frac{\partial^{2} A}{\partial \phi^{2}}-i g\left(|A|^{2} A-f_{\mathrm{R}} \phi_{\mathrm{R}} A \frac{\partial|A|^{2}}{\partial \phi}\right) \\
=\left(-\frac{\kappa}{2}+i\left(\omega_{0}-\omega_{\mathrm{p}}\right)\right) A+\sqrt{\kappa_{\mathrm{ex}}} s_{\mathrm{GSL}}
\end{array}
$$

where $A(\phi, t)$ is the intracavity field envelope, $\omega_{0}$ and $\omega_{\mathrm{p}}$ are the angular frequencies of the pumped resonance and the laser, respectively. The definition of the other parameters and their values can be found in "Methods" and ref. ${ }^{35}$. We define the laserresonance detuning as the frequency difference between the master laser and the corresponding resonance. As shown by the intracavity intensity evolution in Fig. 3c, in the MI regime, solitons are excited around the pulse peak. The formed solitons, however, are pulled to the pulse edges by the phase gradient, until they annihilate when the pumping field amplitude is below the minimum level required for soliton existence.

Next, we simulate the offset-injection-locked GSL by setting the master laser frequency to be $160 \mathrm{GHz}$ lower than the centralmode frequency. With adjusted gain-switching current and injected power, our simulation results presented in Fig. 3d, e 

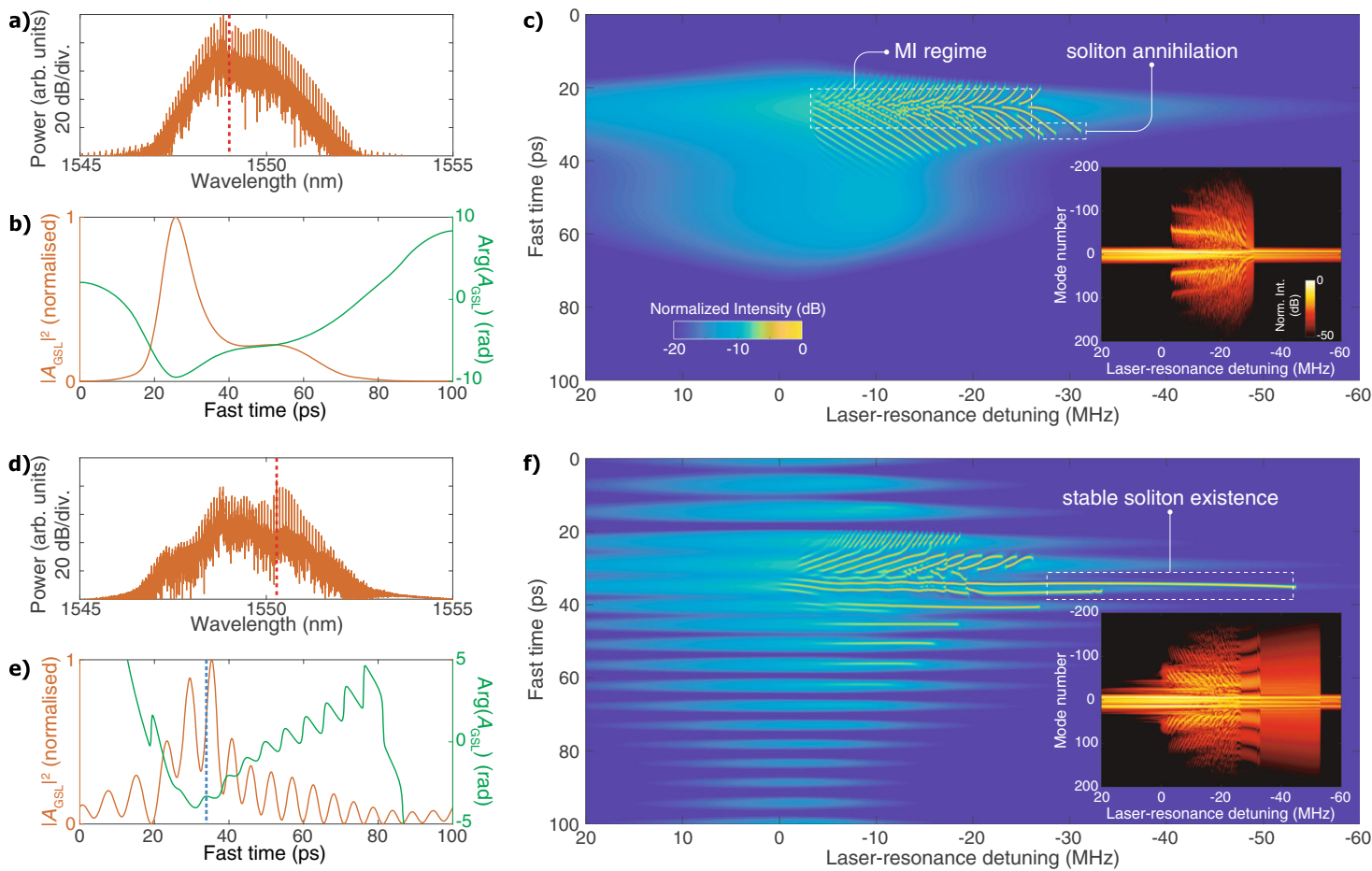

Fig. 3 Simulations of soliton generation with gain-switched laser driving. a Comb spectrum and $\mathbf{b}$ pulse intensity and phase of the gain-switched laser when the master laser is injected into the centre of the lasing mode at $1549 \mathrm{~nm}$. The red dashed line indicates the position of the master laser injection. c Intracavity intensity evolution of a $\mathrm{Si}_{3} \mathrm{~N}_{4}$ microring resonator driven by the GSL pulse in (b). MI modulation instability. The inset shows the microcomb spectrum evolution. d Comb spectrum of the gain-switched laser that is offset-injection-locked. The master laser frequency is detuned from the centre of the intrinsic lasing mode by $-160 \mathrm{GHz}$. e Intensity and phase of the offset-injection-locked GSL pulse. The blue dashed line indicates the phase maximum that has the highest field amplitude. $\mathbf{f}$ Intracavity intensity evolution of the microring resonator that is pumped by the pulse in (e), showing a single-soliton step with a width of $\sim 20 \mathrm{MHz}$ yielded by a soliton trapped at the phase maximum that is indicated by the dashed line in (e).

agrees well with the experiments. This frequency-offset injection successfully excites the simultaneous lasing of two laser modes. While the gain-switched pulses from the two modes have distinct carrier frequencies, they overlap spatiotemporally as they are triggered by the same microwave signal. Remarkably, owing to the interference between the fields of the two constituent pulses (see Supplementary Notes 2 and 3 for more details), the dualmode GSL pulse shows multiple-intensity maxima and local phase maxima. These phase maxima, when their corresponding field amplitudes are strong enough, can trap solitons and facilitate robust soliton microcomb generation. Using the same procedure, we simulate the evolving intracavity field with the multipeak pulse profile, which is shown in Fig. 3f. Indeed, after solitons are triggered by MI, they are stably confined by the phase maxima, despite that the Raman effect tends to shift solitons to locations with a larger fast time. As a result, a wide soliton step is obtained, enabling low-power-threshold DKS with an externally locked $f_{\text {rep. }}$.

Subharmonic pumping with FP GSL. Since FP lasers are intrinsically multimode, one would naturally expect the offsetinjection-locking technique to be applicable with the FP GSL. Due to the limited gain-switching bandwidth of $7 \mathrm{GHz}$ of the FP GSL, we set $f_{\text {gs }}$ to be half of $f_{\text {rep }}$, which is around $4.858 \mathrm{GHz}$. In this way, we can pulse-pump the microresonator subharmonically (see Fig. 4a), with a halved pumping efficiency. The output power of the FP GSL is $4 \mathrm{~mW}$, and amplification is also used. The upper and the lower panels in Fig. $4 \mathrm{~b}$ show the GSL spectra when the master laser is injected around the centre of a mode and offsetinjected to excite multimode lasing, respectively. Again, offsetinjection locking substantially expands the GSL comb bandwidth and creates multiple pulse peaks, shown by the comparison of the measured autocorrelation intensities in Fig. 4c. While we still cannot observe any wide soliton steps with the single-mode-lasing GSL pulses with average power up to $200 \mathrm{~mW}$, we can generate a single-soliton microcomb (whose spectrum is presented in Fig. 4d) by using $40 \mathrm{~mW}$ of the offset-injection-locked GSL. This power level is fourfold larger than that for the DFB GSL because of the subharmonic pumping scheme and the CW component contained in the FP GSL. Nevertheless, the power threshold is lower than that of the CW pumping by nearly a factor of 3 , showing the effectiveness and the flexibility of the GSL in exciting DKSs with repetition rates beyond the gain-switching bandwidth.

Pulse-phase engineering with additional chirping. As recently reported in ref. ${ }^{44}$, when the master laser is detuned from the lasing mode with a relatively large frequency offset, the DM GSL is not injection-locked anymore, and no coherent dual-mode lasing can be excited. This result, however, is not surprising given that the DM laser structure supports only a single lasing mode. To circumvent the difficulty in altering the phase of the DM GSL pulses, we use a waveshaper to apply external chirping. Figure 5a shows the computed pulse intensities and phases before and after the waveshaping with an applied dispersion of $-13 \mathrm{ps} \mathrm{nm}^{-1}$. A local phase maximum is created by the waveshaping, and the pulse width simulated with single-mode laser rate equations is also narrowed. Inserting the phase-engineered pulse into the LLE model with average pumping power of $5 \mathrm{~mW}$, we obtain a remarkably wide $(>40 \mathrm{MHz})$ single-soliton step (see Fig. 5b). Experimentally we apply to chirp on the injection-locked DM GSL, and we tune the dispersion of the waveshaper to observe varied step widths 
a)

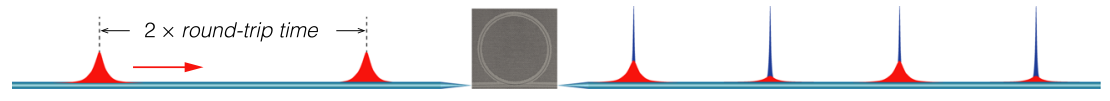

b)

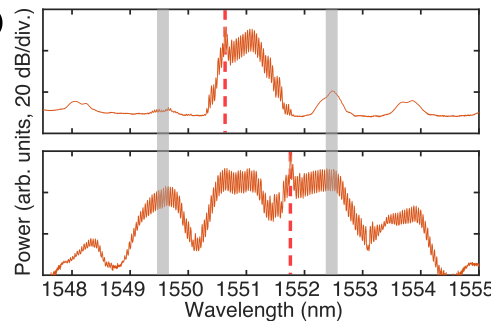

c)

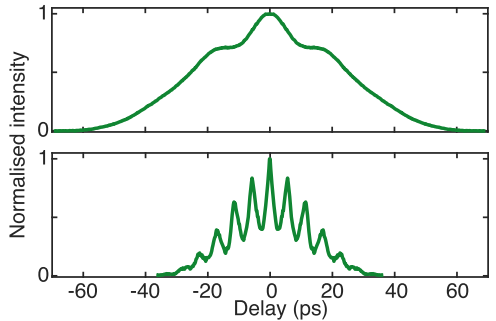

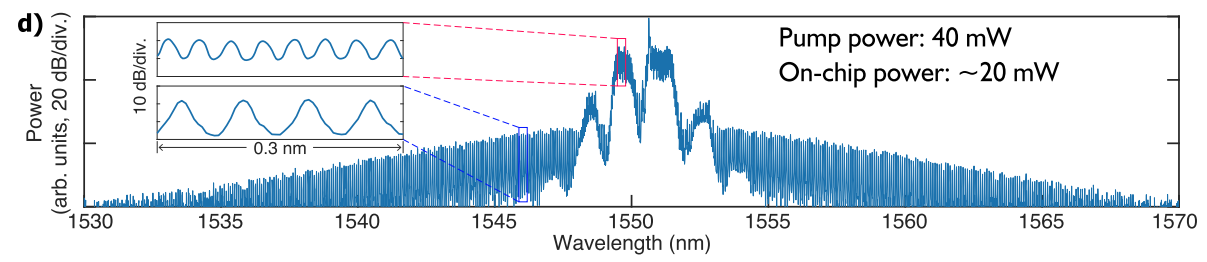

Fig. 4 Subharmonic pumping using a gain-switched Fabry-Perot laser. a Schematic of the subharmonic pulse-pumping method. b GSL spectra obtained with the master laser injecting around the centre of a lasing mode at $1550.7 \mathrm{~nm}$ (upper panel) and between two lasing modes (lower panel), respectively. The grey stripes indicate the positions of the central mode's two neighbouring FP modes. The red dashed lines indicate the positions of the injection. c The measured autocorrelation traces of the GSL pulses in single-mode lasing (upper panel) and dual-mode lasing (lower panel) states, respectively. $\mathbf{d}$ The optical spectrum of the soliton microcomb driven by the offset-injection-locked FP GSL. The insets show the enlarged partial spectra of the GSL comb with $f_{\mathrm{gs}}$ of $4.86 \mathrm{GHz}$ and the microcomb with $f_{\text {rep }}$ of $9.72 \mathrm{GHz}$, respectively.

displayed in Fig. 5c. A dispersion of $-18 \mathrm{ps} \mathrm{nm}^{-1}$ is found to yield the maximum step width, not only showing good agreement with the computation but also allowing us to generate a soliton microcomb with an average pump power of $10 \mathrm{~mW}$ (see Fig. $5 \mathrm{~d}$ ). With a high biased current of $120 \mathrm{~mA}$, the averaged output power of the DM GSL is $13 \mathrm{~mW}$, which, in principle, could generate solitons in the $\mathrm{Si}_{3} \mathrm{~N}_{4}$ microresonators without additional amplification. However, due to the significant $(>4 \mathrm{~dB})$ loss induced by the waveshaper, amplification is still needed in this proof-of-principle experiment. Nevertheless, the demonstrated power threshold that is below the output power of the DM GSL shows promise for direct pulse-driven soliton generation if integrated dispersion compensation $^{45}$ is implemented.

\section{Discussion}

A gain-switching technique is implemented to generate lowrepetition-rate soliton microcombs with semiconductor laser pulses. Phase alteration of the laser pulses, either using the dualmode lasing state or applying auxiliary waveshaping, turns unfavourable intracavity laser field profiles into effective soliton traps that are necessary for the robust soliton generation and the flexible switching of microcomb states (see Supplementary Note 4 for details). The crucial role of the phase profile of the driving pulses could also have vital implications to soliton generation with advanced pulsed laser sources such as mode-locked quantum dot lasers $^{46}$. Based on the fusion of two different compact frequency comb technologies, our work shows the prospect of using only chip-based components to generate energy-efficient wideband frequency combs, which could open the door for field-deployable applications of compact frequency combs in microwave photonics, sensing and metrology. Although the gain-switching scheme needs a microwave source, which potentially increases the complexity of the system, the flexible control and stabilisation of the soliton repetition rate is particularly beneficial to applications that require a fixed repetition rate and a rapidly tunable carrier frequency, such as frequency-modulated coherent Lidar ${ }^{19}$.
Besides the significance to the development of practical microcomb sources, our work can be found useful for the study of complex laser dynamics ${ }^{10}$ and fundamental cavity soliton physics such as the spontaneous symmetry breaking and trapping of solitons by periodically modulated driving fields ${ }^{47-50}$ and the nonlinear filtering effect in dissipative solitons ${ }^{51}$.

\section{Methods}

Gain-switched semiconductor lasers. The DFB laser is manufactured by NTT Electronics. It is contained within a hermetically sealed high-speed package. The 7pin package is incorporated with a high-speed rf input to enable direct modulation. The gain-switching bandwidth of the laser is up to $20 \mathrm{GHz}$. The typical bias current is set between 40 and $60 \mathrm{~mA}$.

The FP laser and the DM laser are commercially available from Eblana Photonics. They are packaged in high-speed butterfly packages. The FP laser has an effective gain-switching bandwidth of $7 \mathrm{GHz}$, and the applied bias current is around $60 \mathrm{~mA}$. The DM laser can be gain-switched with a frequency up to $14 \mathrm{GHz}$. It can be operated with a high bias current above $120 \mathrm{~mA}$. Compared with the DFB laser, the DM laser shows an SMSR that is higher by $7 \mathrm{~dB}$, although its temperature-dependent wavelength tuning range is reduced to approximately 2 $\mathrm{nm}$, which is adequate for pumping microresonators with FSRs that are less than $250 \mathrm{GHz}$.

To activate the gain-switching mode, a microwave tone derived from a microwave synthesiser (Rohde\&Schwarz SMB100A) is amplified and combined through a bias tee with the DC current. The power level of the microwave is typically between 18 and $26 \mathrm{dBm}$. To injection-lock the GSLs, an injected power around $1 \mathrm{~mW}$ from the ECDL (Toptica Photonics) is sent into the GSLs via a fibre circulator. An erbium-doped fibre amplifier (Keopsys, the maximum output power of $33 \mathrm{dBm}$ ) is utilised to amplify the GSL power for soliton pumping. We found that the pump laser linewidth usually needs to be much narrower than the microresonator mode bandwidth to reliably generate solitons, which is reasonable as only a narrower laser linewidth would allow the laser power to be efficiently accumulated in the resonator. This explains why compact semiconductor lasers usually need master laser injection locking or self-injection locking to assist in soliton microcomb generation. However, in order to fully understand the tolerable laser linewidth, a quantitative study on the laser-frequency noise spectrum's impact on soliton stability is to be conducted in the future.

Microresonators. The $\mathrm{MgF}_{2}$ WGMR is made from a $\mathrm{z}$-cut crystalline rod with diamond shaping and surface polishing. A tapered fibre is used to couple light into the resonator. The loaded quality factor $(Q)$ of the mode resonance that generates 
a)

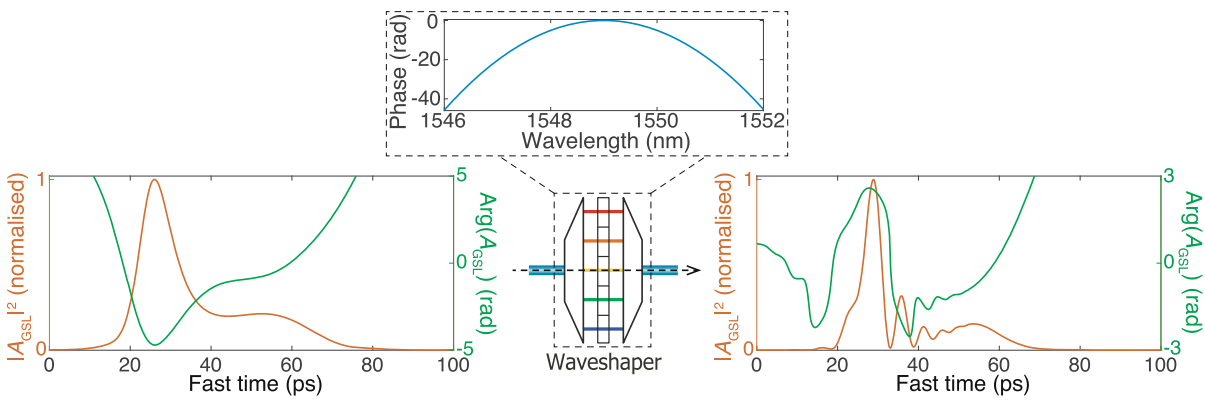

b)
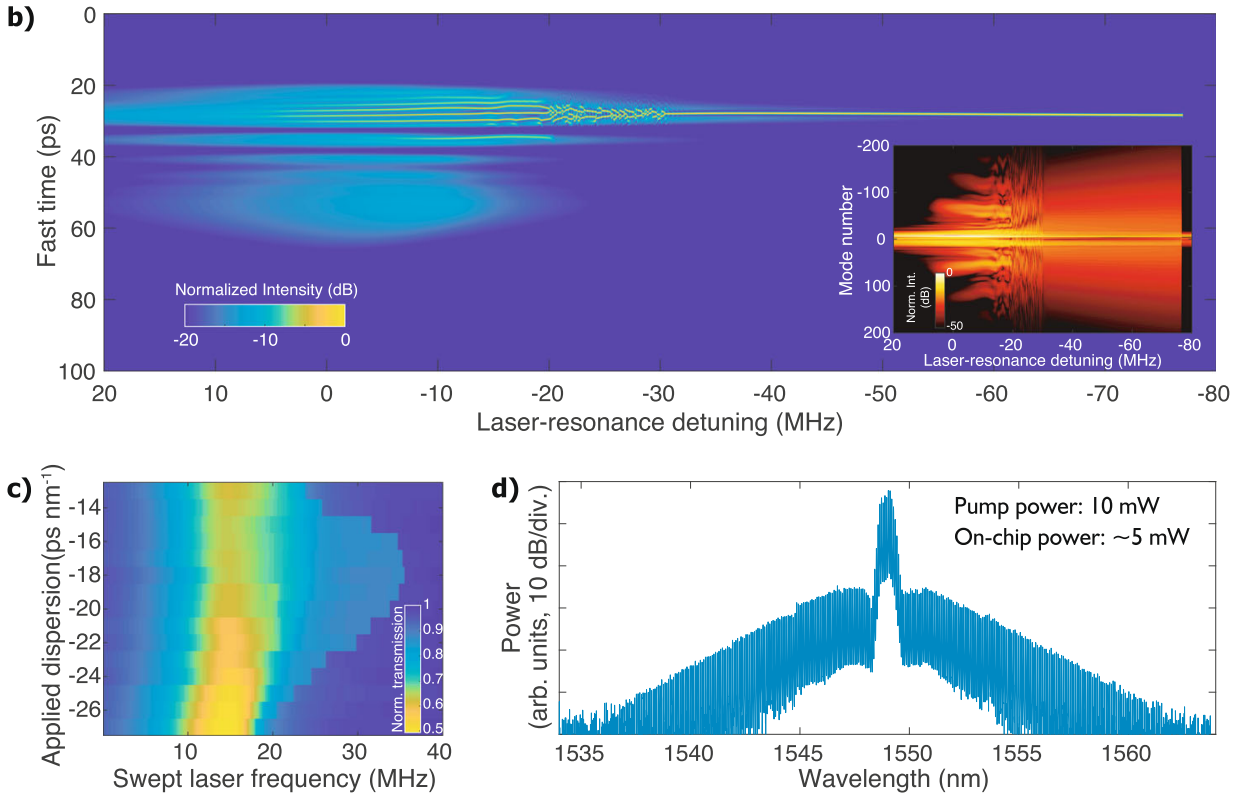

Fig. 5 Importance of phase engineering in pulse pumping with a gain-switched discrete mode laser. a Auxiliary dispersion is applied to the simulated GSL pulse in order to achieve a phase maximum around the intensity peak. b Simulated soliton generation with externally chirped GSL pulses. The comb spectrum evolution is displayed in the inset. c Contour plot of the laser-frequency-swept transmission using the DM GSL with varied applied dispersion. d Single-soliton microcomb spectrum experimentally generated with $10 \mathrm{~mW}$ of the DM GSL power using an external dispersion of $-18 \mathrm{ps} n \mathrm{~m}^{-1}$.

combs is $\sim 1 \times 10^{9}$, corresponding to a resonance bandwidth $\left(\frac{\kappa}{2 \pi}\right)$ of $200 \mathrm{kHz}$. The dispersion parameter $\left(D_{2}\right)$ is approximately $2 \pi \times 2 \mathrm{kHz}$.

The $\mathrm{Si}_{3} \mathrm{~N}_{4}$ microresonator was fabricated with the Photonic Damascene Process ${ }^{52,53}$. The coupling section is designed to operate close to the critical coupling regime for $1550 \mathrm{~nm}$. The loaded resonance bandwidth is around $20 \mathrm{MHz}$, corresponding to a loaded $Q$ of $1 \times 10^{7}$. The dispersion coefficient $D_{2}$ is measured to be $2 \pi \times 15 \mathrm{kHz}$. Lensed fibres are used to couple light into and out of the nanotapered waveguide ${ }^{54}$.

GSL simulations. The numerical simulation is carried out with the Runge-Kutta method. When the master laser injection is presented, the spontaneous emission is not necessary for simulating the GSL pulse profiles. However, we include this effect to obtain the GSL comb spectra with noise floors shown in Fig. 3. The noise terms are expressed as $F_{\mathrm{A}}=$ $\sqrt{\beta \gamma_{2} N^{2} B_{\text {sim }}}\left(e_{\mathrm{I}}(t)+i e_{\mathrm{Q}}(t)\right)$ and $F_{\mathrm{N}}=\sqrt{2\left(\gamma_{1} N+\gamma_{2} N^{2}+\gamma_{3} N^{3}\right) B_{\text {sim }}} e_{\mathrm{N}}(t)$. Here, $\beta=1 \times 10^{-4}$ is a factor related to the amount of spontaneous emission into the lasing mode, $B_{\text {sim }}=5 \times 10^{12} \mathrm{~Hz}$ is the simulation bandwidth. The stochastic unit Gaussian random variable noise terms $e_{\mathrm{N}}, e_{\mathrm{I}}$ and $e_{\mathrm{Q}}$ have zero mean value and unity variance. For the optical field, both the in-phase component $e_{\mathrm{I}}$ and the quadrature component $e_{\mathrm{Q}}$ are included.

For the GSL simulations, we use 4.5 for $\alpha_{\mathrm{H}}, 4.5 \times 10^{4} \mathrm{~s}^{-1}$ for $a, 1 \times 10^{24} \mathrm{~m}^{-3}$ for $N_{0}, 2.5 \mathrm{ps}$ for $\tau_{\mathrm{P} 1}, 1.4 \mathrm{ps}$ for $\tau_{\mathrm{P} 2}$ and $5 \times 10^{-17} \mathrm{~m}^{3}$ for $V$. For the carrier recombination rate, $\gamma_{1}$ is $1 \times 10^{9} \mathrm{~s}^{-1}, \gamma_{2}$ is $1 \times 10^{-16} \mathrm{~s}^{-1} \mathrm{~m}^{3}$ and $\gamma_{3}$ is $1 \times 10^{-41} \mathrm{~s}^{-1} \mathrm{~m}^{6}$. To simulate the central-mode-injected GSL dynamics, $I_{\text {bias }}$ and $I_{\mathrm{gs}}$ are both set to be $75 \mathrm{~mA}$, and the source term $\sqrt{\kappa_{\mathrm{c}}} s_{\text {inj }}$ is $4 \times 10^{21} \mathrm{~m}^{-3 / 2} \mathrm{~s}^{-1}$. For the offset-injection-locking scenario, $I_{\text {bias }}$ is $75 \mathrm{~mA}, I_{\mathrm{gs}}$ is $55 \mathrm{~mA}$ and $\sqrt{\kappa_{\mathrm{c}}} s_{\text {inj }}$ is $3.28 \times 10^{22} \mathrm{~m}^{-3 / 2} \mathrm{~s}^{-1}$. We note that the set of parameter values we use are not for a particular GSL type in the experiments. Instead, they are chosen to be within the reasonable ranges of typical parameter values so that the model is general and qualitative. For example, the linewidth enhancement factor (also known as the Henry factor) is set to be 4.5 because usually it is between 2 and 7 , and for the DFB laser, it was measured to be around $5^{5}$. To qualitatively reproduce the gain-switching behaviours, one can vary the values of multiple parameters and obtain similar results.

Because it is the relative phase of the pumping field that matters in the soliton pumping, all the simulated GSL-phase profiles presented in this work are offsetadjusted to be centred around the 0 -radian level.

Pulse-driven soliton generation simulations. Some parameters in Eq. (4) are defined as such $D_{2}$ is the second-order dispersion coefficient, $\phi$ is the corotating angular coordinate that is related to the round-trip fast time coordinate $\tau$ by $\phi=$ $\tau \times D_{1}$ (where $\frac{D_{1}}{2 \pi}$ is the FSR), $\kappa_{\mathrm{ex}}$ is the external coupling rate, $\kappa$ is the cavity decay rate that includes both the external coupling rate and the intrinsic loss rate caused by mechanisms such as material absorption and roughness-induced scattering (which is the dominant intrinsic loss mechanism in the microresonators in this work) and $s_{\mathrm{GSL}}$ is the input GSL field. In the LLE, $g$ is the single-photon-induced Kerr frequency shift, $f_{\mathrm{R}}$ is the Raman fraction and $\phi_{\mathrm{R}}$ is the term related to the Raman shock time ${ }^{35}$.

To simulate the soliton generation with Eq. (4), the GSL pulse profiles are used as the pumping term after proper interpolation is performed to match the temporal resolution of the LLE simulation. The FSR of the resonator is set to be $10 \mathrm{GHz}$, the second-order dispersion coefficient $D_{2}$ is $2 \pi \times 15 \mathrm{kHz}$ and $g$ is $0.19 \mathrm{~Hz}$. The cavity decay rate is $\kappa=2 \pi \times 20 \mathrm{MHz}$, and $\kappa_{\mathrm{ex}}$ is set to be equal to $\frac{\kappa}{2}$. To include the Raman effect, $f_{\mathrm{R}}=0.008$ and $\phi_{\mathrm{R}}=2 \times 10^{-4} \mathrm{rad}$ are used. For all the simulations shown in Figs. 3 and 5, the laser sweeping speed is $4 \times 10^{12} \mathrm{~Hz} \mathrm{~s}^{-1}$.

Autocorrelation measurement. To measure the autocorrelation intensities of the GSL pulses, the GSL light is split interferometrically and recombined with an adjustable delay in a barium borate crystal to generate the second harmonic signal in a noncollinear fashion. The intensity of the second harmonic signal is continuously measured as the delay is scanned. Due to the limited maximum delay of 
the setup, the measurement can only cover more than half of the full delay range for the FP GSL pulses whose repetition rate is relatively low. Thanks to the symmetrical nature of the autocorrelation traces, we horizontally flip the partial autocorrelation traces with respect to the positions of the intensity maxima to produce the full traces shown in Fig. $4 \mathrm{c}$.

\section{Data availability}

The data used to produce the results of this paper are available on Zenodo.

\section{Code availability}

The code that supports the plots within this paper is available on Zenodo.

Received: 21 July 2020; Accepted: 22 January 2021;

Published online: 03 March 2021

\section{References}

1. Tarucha, S. \& Otsuka, K. Response of semiconductor laser to deep sinusoidal injection current modulation. IEEE J. Quantum Electronics 17, 810-816 (1981).

2. Ziel, J. Vander \& Logan, R. Generation of short optical pulses in semiconductor lasers by combined dc and microwave current injection. IEEE J. Quantum Electronics 18, 1340-1350 (1982).

3. Paulus, P., Langenhorst, R. \& Jager, D. Generation and optimum control of picosecond optical pulses from gain-switched semiconductor lasers. IEEE J. Quantum Electronics 24, 1519-1523 (1988).

4. Zhou, R. et al. $40 \mathrm{~nm}$ wavelength tunable gain-switched optical comb source. Opt. Express 19, B415-B420 (2011).

5. Anandarajah, P. M. et al. Generation of coherent multicarrier signals by gain switching of discrete mode lasers. IEEE Photonics J. 3, 112-122 (2011).

6. Herbert, C. et al. Discrete mode lasers for communication applications. IET Optoelectronics 3, 1-17 (2009).

7. Imran, M., Anandarajah, P. M., Kaszubowska-Anandarajah, A., Sambo, N. \& Potí, L. A survey of optical carrier generation techniques for terabit capacity elastic optical networks. IEEE Commun. Surv. Tutor. 20, 211-263 (2017).

8. Jofre, M. et al. True random numbers from amplified quantum vacuum. Optics Express 19, 20665-20672 (2011).

9. Yuan, Z. L. et al. Interference of short optical pulses from independent gainswitched laser diodes for quantum secure communications. Phys. Rev. Appl. 2, 064006 (2014).

10. Yuan, Z. L. et al. Directly phase-modulated light source. Phys. Rev. X 6, 031044 (2016).

11. Marpaung, D., Yao, J. \& Capmany, José Integrated microwave photonics. Nat. Photonics 13, 80-90 (2019).

12. Tang, Z., Li, Y., Yao, J. \& Pan, S. Photonics-based microwave frequency mixing: methodology and applications. Laser Photonics Rev. 14, 1800350 (2020).

13. Cortés, L. R., Onori, D., de Chatellus, H. G., Burla, M. \& Azaña, J. Towards on-chip photonic-assisted radio-frequency spectral measurement and monitoring. Optica 7, 434-447 (2020).

14. Kippenberg, T. J., Gaeta, A. L., Lipson, M. \& Gorodetsky, M. L. Dissipative kerr solitons in optical microresonators. Science 361, eaan8083 (2018).

15. Gaeta, A. L., Lipson, M. \& Kippenberg, T. J. Photonic-chip-based frequency combs. Nat. Photonics 13, 158-169 (2019).

16. Marin-Palomo, P. et al. Microresonator-based solitons for massively parallel coherent optical communications. Nature 546, 274-279 (2017).

17. Trocha, P. et al. Ultrafast optical ranging using microresonator soliton frequency combs. Science 359, 887-891 (2018).

18. Suh, M.-G. \& Vahala, K. J. Soliton microcomb range measurement. Science 359, 884-887 (2018a).

19. Riemensberger, J. et al. Massively parallel coherent laser ranging using a soliton microcomb. Nature 581, 164-170 (2020).

20. Stern, B., Ji, X., Okawachi, Y., Gaeta, A. L. \& Lipson, M. Battery-operated integrated frequency comb generator. Nature 562, 401-405 (2018).

21. Raja, A. S. et al. Electrically pumped photonic integrated soliton microcomb. Nat. Commun. 10, 1-8 (2019).

22. Shen, B. et al. Integrated turnkey soliton microcombs. Nature 582, 365-369 (2020).

23. Chang, L. et al. Ultra-efficient frequency comb generation in algaas-oninsulator microresonators. Nat. Commun. 11, 1-8 (2020).

24. Suh, M.-G. \& Vahala, K. Gigahertz-repetition-rate soliton microcombs. Optica 5, 65-66 (2018b)

25. Suh, M.-G., Wang, C. Y., Johnson, C. \& Vahala, K. J. Directly pumped $10 \mathrm{ghz}$ microcomb modules from low-power diode lasers. Opt. Lett. 44, 1841-1843 (2019).
26. Liu, J. et al. Photonic microwave generation in the $\mathrm{x}$ - and $\mathrm{k}$-band using integrated soliton microcombs. Nat. Photonics (2020).

27. Obrzud, E., Lecomte, S. \& Herr, T. Temporal solitons in microresonators driven by optical pulses. Nat. Photonics 11, 600 (2017).

28. Obrzud, E. et al. A microphotonic astrocomb. Nat. Photonics 13, 31-35 (2019).

29. Murata, H., Morimoto, A., Kobayashi, T. \& Yamamoto, S. Optical pulse generation by electrooptic-modulation method and its application to integrated ultrashort pulse generators. IEEE J. Select. Top. Quantum Electronics 6, 1325-1331 (2000)

30. Weng, W. et al. Spectral purification of microwave signals with disciplined dissipative kerr solitons. Phys. Rev. Lett. 122, 013902 (2019).

31. Liang, W. et al. High spectral purity kerr frequency comb radio frequency photonic oscillator. Nat. Commun. 6, 7957 (2015).

32. Pavlov, N. G. et al. Narrow-linewidth lasing and soliton kerr microcombs with ordinary laser diodes. Nat. Photonics 12, 694-698 (2018).

33. Bao, C. et al. Soliton repetition rate in a silicon-nitride microresonator. Opt Lett. 42, 759-762 (2017).

34. Yi, X., Yang, Q.-F., Yang, K. Y. \& Vahala, K. Theory and measurement of the soliton self-frequency shift and efficiency in optical microcavities. Opt. Lett. 41, 3419-3422 (2016)

35. Karpov, M. et al. Raman self-frequency shift of dissipative kerr solitons in an optical microresonator. Phys. Rev. Lett. 116, 103902 (2016).

36. Ó Dúill, S. P., Anandarajah, P. M., Zhou, R. \& Barry, L. P. Numerical investigation into the injection-locking phenomena of gain switched lasers for optical frequency comb generation. Applied Physics Letters 106, 211105 (2015)

37. Schunk, N. \& Petermann, K. Noise analysis of injection-locked semiconductor injection lasers. IEEE J. Quantum Electronics 22, 642-650 (1986).

38. Luo, K., Jang, J. K., Coen, S., Murdoch, S. G. \& Erkintalo, M. Spontaneous creation and annihilation of temporal cavity solitons in a coherently driven passive fiber resonator. Opt. Lett. 40, 3735-3738 (2015).

39. Jang, J. K., Erkintalo, M., Coen, S. \& Murdoch, S. G. Temporal tweezing of light through the trapping and manipulation of temporal cavity solitons. Nat. Commun. 6, 7370 (2015).

40. Taheri, H., Eftekhar, A. A., Wiesenfeld, K. \& Adibi, A. Soliton formation in whispering-gallery-mode resonators via input phase modulation. IEEE Photonics J. 7, 1-9 (2015).

41. Godey, C., Balakireva, I. V., Coillet, A. \& Chembo, Y. K. Stability analysis of the spatiotemporal lugiato-lefever model for kerr optical frequency combs in the anomalous and normal dispersion regimes. Phys. Rev. A 89, 063814 (2014)

42. Lugiato, L. A. \& Lefever, R. Spatial dissipative structures in passive optical systems. Phys. Rev. Lett. 58, 2209 (1987).

43. Haelterman, M., Trillo, S. \& Wabnitz, S. Dissipative modulation instability in a nonlinear dispersive ring cavity. Opt. Commun. 91, 401-407 (1992)

44. Rosado, A. et al. Numerical and experimental analysis of optical frequency comb generation in gain-switched semiconductor lasers. IEEE J. Quantum Electronics 55, 1-12 (2019).

45. Madsen, C. K. et al. Integrated all-pass filters for tunable dispersion and dispersion slope compensation. IEEE Photonics Technol. Lett. 11, 1623-1625 (1999).

46. Rafailov, E. U., Cataluna, M. A. \& Sibbett, W. Mode-locked quantum-dot lasers. Nat. Photonics 1, 395-401 (2007).

47. Hendry, I. et al. Spontaneous symmetry breaking and trapping of temporal kerr cavity solitons by pulsed or amplitude-modulated driving fields. Phys. Rev. A 97, 053834 (2018)

48. Taheri, H., Matsko, A. B. \& Maleki, L. Optical lattice trap for kerr solitons. Eur. Phys. J. D 71, 153 (2017)

49. Xue, X., Zheng, X. \& Weiner, A. M. Soliton trapping and comb selfreferencing in a single microresonator with $\chi(2)$ and $\chi(3)$ nonlinearities. Opt. Lett. 42, 4147-4150 (2017).

50. Cole, D. C. et al. Kerr-microresonator solitons from a chirped background. Optica 5, 1304-1310 (2018).

51. Brasch, V., Obrzud, E., Lecomte, S. \& Herr, T. Nonlinear filtering of an optica pulse train using dissipative kerr solitons. Optica 6, 1386-1393 (2019).

52. Pfeiffer, M. H. P. et al. Photonic damascene process for integrated high-q microresonator based nonlinear photonics. Optica 3, 20-25 (2016).

53. Liu, J. et al. Ultralow-power chip-based soliton microcombs for photonic integration. Optica 5, 1347-1353 (2018a).

54. Liu, J. et al. Double inverse nanotapers for efficient light coupling to integrated photonic devices. Opt. Lett. 43, 3200-3203 (2018b).

\section{Acknowledgements}

W.W. thanks Romain Bouchand, Miles Anderson, Arslan Raja and Johann Riemensberger for technical assistance, and Cherise M. Qiu for assistance in illustration. The authors acknowledge the assistance from Rui Ning Wang on silicon nitride resonator fabrication. This publication was supported by Contract No. D18AC00032 (DRINQS) from the Defense Advanced Research Projects Agency (DARPA), Defense Sciences Office 
(DSO), funding from the Swiss National Science Foundation under grant agreement No. 192293, Science Foundation Ireland (SFI) 15/CDA/3640 and the SFI/European Regional Development Fund (13/RC/2077). This work is also supported by the Air Force Office of Scientific Research under award number FA9550-19-1-0250.

\section{Author contributions}

W.W., A.K.-A. and P.M.A. conceived the project. W.W. performed the experiments with A.K.-A., J.H. and E.L.'s assistance. W.W. analysed the data, developed the theoretical model and performed the numerical simulations. A.K.-A., P.D.L. and P.M.A. developed and tested the gain-switched lasers. J.L. designed and fabricated the silicon nitride resonator. J.H. and J.L. characterised the silicon nitride resonator. W.W. wrote the paper, with input from other authors. P.M.A. and T.J.K. supervised the project.

\section{Competing interests}

The authors declare no competing interests.

\section{Additional information}

Supplementary information The online version contains supplementary material available at https://doi.org/10.1038/s41467-021-21569-7.

Correspondence and requests for materials should be addressed to W.W., P.M.A. or T.J.K.
Peer review information Nature Communications thanks Roberto Llorente Sáez, Stefan Breuer and the other anonymous reviewer for their contribution to the peer review of this work.

Reprints and permission information is available at http://www.nature.com/reprints

Publisher's note Springer Nature remains neutral with regard to jurisdictional claims in published maps and institutional affiliations.

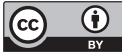

Open Access This article is licensed under a Creative Commons Attribution 4.0 International License, which permits use, sharing, adaptation, distribution and reproduction in any medium or format, as long as you give appropriate credit to the original author(s) and the source, provide a link to the Creative Commons license, and indicate if changes were made. The images or other third party material in this article are included in the article's Creative Commons license, unless indicated otherwise in a credit line to the material. If material is not included in the article's Creative Commons license and your intended use is not permitted by statutory regulation or exceeds the permitted use, you will need to obtain permission directly from the copyrigh holder. To view a copy of this license, visit http://creativecommons.org/licenses/by/4.0/.

(C) The Author(s) 2021 